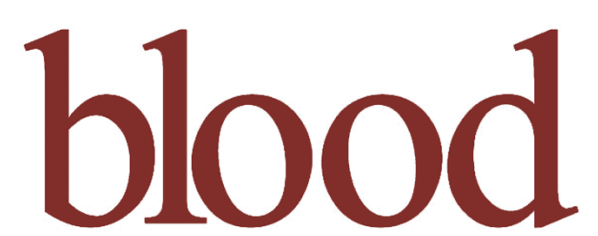

2011 117: 5620-5630

Prepublished online February 25, 2011;

doi:10.1182/blood-2010-08-300236

\title{
The EMT regulator Zeb2/Sip1 is essential for murine embryonic hematopoietic stem/progenitor cell differentiation and mobilization
}

Steven Goossens, Viktor Janzen, Sonia Bartunkova, Tomomasa Yokomizo, Benjamin Drogat, Mihaela Crisan, Katharina Haigh, Eve Seuntjens, Lieve Umans, Tamara Riedt, Pieter Bogaert, Lieven Haenebalcke, Geert Berx, Elaine Dzierzak, Danny Huylebroeck and Jody J. Haigh

Updated information and services can be found at:

http://bloodjournal.hematologylibrary.org/content/117/21/5620.full.html

Articles on similar topics can be found in the following Blood collections

Hematopoiesis and Stem Cells (3057 articles)

Information about reproducing this article in parts or in its entirety may be found online at:

http://bloodjournal.hematologylibrary.org/site/misc/rights.xhtml\#repub_requests

Information about ordering reprints may be found online at:

http://bloodjournal.hematologylibrary.org/site/misc/rights.xhtml\#reprints

Information about subscriptions and ASH membership may be found online at: http://bloodjournal.hematologylibrary.org/site/subscriptions/index.xhtml

Blood (print ISSN 0006-4971, online ISSN 1528-0020), is published weekly by the American Society of Hematology, 2021 L St, NW, Suite 900, Washington DC 20036.

Copyright 2011 by The American Society of Hematology; all rights reserved.

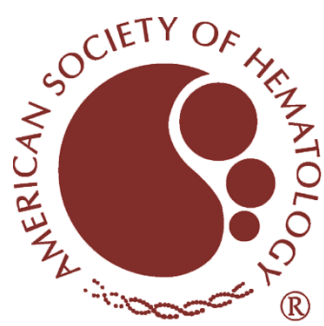




\title{
The EMT regulator Zeb2/Sip1 is essential for murine embryonic hematopoietic stem/progenitor cell differentiation and mobilization
}

\author{
*Steven Goossens, ${ }^{1,2}$ *Viktor Janzen, ${ }^{3}$ Sonia Bartunkova, ${ }^{1,2}$ Tomomasa Yokomizo, ${ }^{4}$ Benjamin Drogat, ${ }^{1,2}$ Mihaela Crisan, ${ }^{4}$ \\ Katharina Haigh, ${ }^{1,2}$ Eve Seuntjens, ${ }^{5,6}$ Lieve Umans, 5,6 Tamara Riedt, ${ }^{3}$ Pieter Bogaert, ${ }^{2}$ Lieven Haenebalcke, ${ }^{1,2}$ Geert Berx, ${ }^{2,7}$ \\ Elaine Dzierzak, ${ }^{4}{ }^{*}$ Danny Huylebroeck, ${ }^{5,6}$ and *Jody J. Haigh ${ }^{1,2}$ \\ ${ }^{1}$ Vascular Cell Biology Unit, Department for Molecular Biomedical Research, VIB, Ghent, Belgium; 2Department for Molecular Biomedical Research, Ghent \\ University, Ghent, Belgium; ${ }^{3}$ Department of Internal Medicine III, Hematology and Oncology, University of Bonn, Germany; ${ }^{4}$ Erasmus MC Stem Cell Institute, \\ Department of Cell Biology, Erasmus University Medical Center, Rotterdam, The Netherlands; ${ }^{5}$ Laboratory of Molecular Biology (Celgen), Center for Human \\ Genetics (CME), Katholieke Universiteit Leuven (KULeuven), Leuven, Belgium; ${ }^{6} \mathrm{VIB}$ Department of Molecular and Developmental Genetics, Leuven, Belgium; \\ and ${ }^{7}$ Molecular Oncology Unit, VIB Department for Molecular Biomedical Research, Ghent, Belgium
}

Zeb2 (Sip1/Zfhx1b) is a member of the zinc-finger E-box-binding (ZEB) family of transcriptional repressors previously demonstrated to regulate epithelial-tomesenchymal transition (EMT) processes during embryogenesis and tumor progression. We found high Zeb2 mRNA expression levels in HSCs and hematopoietic progenitor cells (HPCs), and examined Zeb2 function in hematopoiesis through a conditional deletion approach using the Tie2-Cre and Vav-iCre recombi- nation mouse lines. Detailed cellular analysis demonstrated that Zeb2 is dispensable for hematopoietic cluster and HSC formation in the aorta-gonadomesonephros region of the embryo, but is essential for normal HSC/HPC differentiation. In addition, Zeb2-deficient HSCs/HPCs fail to properly colonize the fetal liver and/or bone marrow and show enhanced adhesive properties associated with increased $\beta 1$ integrin and Cxcr4 expression. Moreover, deletion of Zeb2 resulted in embry- onic (Tie2-Cre) and perinatal (Vav-icre) lethality due to severe cephalic hemorrhaging and decreased levels of angiopoietin-1 and, subsequently, improper pericyte coverage of the cephalic vasculature. These results reveal essential roles for Zeb2 in embryonic hematopoiesis and are suggestive of a role for Zeb2 in hematopoietic-related pathologies in the adult. (Blood. 2011;117(21):5620-5630)

\section{Introduction}

Zeb2 (also known as Sip1 and Zfhx1b) is a DNA-binding transcriptional regulator of the family of zinc-finger E-box-binding (ZEB) proteins. ${ }^{1,2}$ Its expression and functioning during development have been associated with epithelial-to-mesenchymal transitions (EMTs). ${ }^{3}$ EMTs encompass a series of events in which polarized epithelial cells become round in shape, lose their cell-cell contacts, and acquire the motile, migratory properties of mesenchymal cells. ${ }^{4}$ This physiologic process is essential for many developmental processes, including mesoderm formation during gastrulation and neural crest delamination and migration. Similar EMT-like changes in cellular morphology can be observed during tumor progression, allowing tumor cells to acquire the capacity to invade the surrounding tissue and ultimately metastasize to a distant site. Subsequent tissue colonization occurs via a reverse transitional mechanism called the mesenchymal-to-epithelial transition. Given the importance of EMT and the mesenchymal-to-epithelial transition in developmental processes and disease, numerous studies have identified several EMT-inducing or EMT-regulating transcription factors, including Zeb2. ${ }^{5}$

Recent studies of neoplastic tissues have demonstrated the existence of cancer stem cells (CSCs), tumor-initiating cells with a self-renewal capacity that exhibit an ability to induce new tumors when transplanted into nude and/or syngeneic mouse strains. ${ }^{6}$ The existence of CSCs was initially discovered in leukemia samples, but they have also been identified in various solid tumor types. The origin of CSCs was until now unclear, but compelling results from Mani et $\mathrm{al}^{7}$ now link EMT processes with the formation of CSCs. EMT induction in an immortalized human mammary epithelial cell line resulted in the acquisition of mesenchymal traits, the expression of stem cell markers, and an enhanced capacity to form mammospheres, a property previously and exclusively associated with mammary epithelial stem cells. ${ }^{7}$ Suppression of the miR-200 family members, which together with miR-205 have previously been shown to negatively regulate Zeb family members, ${ }^{8}$ not only elevates Zeb1 and/or Zeb2 expression, but also several stem cell factors (including Bmi), resulting in increased stemness and metastasisinitiating capacity. ${ }^{9}$ These findings illustrate a potential direct link between EMT induction and the acquisition of stem cell properties.

It is in this context that we analyzed the role of the EMT inducer Zeb2 in the formation of tissue-specific stem cells in vivo, specifically within the hematopoietic system. Mature blood cells arise from HSCs that are capable of generating every hematopoietic cell type, including the various lymphoid and myeloid lineages. Each HSC has the capacity to generate large numbers of mature hematopoietic cells throughout its life, and the HSC pool size in adults is regulated by finely tuned self-renewal and differentiation
Submitted August 6, 2010; accepted January 31, 2011. Prepublished online as Blood First Edition paper, February 25, 2011; DOI 10.1182/blood-201008-300236.

*S.G., V.J., D.H., and J.J.H. contributed equally to this study.
The online version of this article contains a data supplement.

The publication costs of this article were defrayed in part by page charge payment. Therefore, and solely to indicate this fact, this article is hereby marked "advertisement" in accordance with 18 USC section 1734.

(C) 2011 by The American Society of Hematology 
programs controlled by essential transcriptional regulatory networks. ${ }^{10}$ HSCs arise during mammalian embryogenesis in a complex developmental process and migrate to several sequential anatomical sites, ${ }^{11}$ including the yolk sac, the aorta-gonadomesonephros (AGM) region, placenta, fetal liver, and finally the bone marrow in the adult. The first definitive adult-type HSCs in the mouse are generated in the aorta at embryonic day 10.5 $(E 10.5)^{12}$ from hemogenic endothelial cells in the ventral wall of the dorsal aorta, and are part of the hematopoietic clusters that are closely associated with the endothelium. Hematopoietic cluster formation involves changes in cell shape and loss of adhesion from the hemogenic endothelium, ${ }^{13}$ which is similar to an EMT process. This observation, together with the abundant expression of Zeb2 in HSCs, prompted us to analyze its role in hematopoiesis. Conditional deletion of Zeb2 in the hematopoietic system using 2 distinct Cre lines (Tie2-Cre and Vav-iCre) demonstrated that Zeb2 is not involved in the initial formation of HSCs, but is essential for their subsequent differentiation and mobilization. These data indicate key roles for Zeb2 in definitive embryonic hematopoiesis and provide new cellular and genetic evidence for a physiologic role of EMT regulators in HSC differentiation and mobilization.

\section{Methods}

\section{Mouse genetic intercrosses}

The generation of the conditional (with floxed critical exon 7, here referred to as fl) and total (globally deleted critical exon7, here referred to as a null or -) Zeb2-knockout alleles was described previously. ${ }^{14,15}$ To achieve high penetrance of the phenotype, we intercrossed heterozygote conventional mutants $(-/+)$ with the conditional (fl/fl) strain. The same breeding strategy was used for transgenic (tg) Tie2-cre- and Vav-iCre-mediated Zeb2 deletion. Tie2-Cre ${ }^{\mathrm{tg} /+} ; \mathrm{Zeb} 2^{-/+}$or Vav-iCre ${ }^{\mathrm{tg} /+} ; \mathrm{Zeb} 2^{-/+}$males were crossed with Zeb2 $2^{\mathrm{fl} / \mathrm{fl}} ; \mathrm{R} 26-\mathrm{LacZ} \mathrm{Z}^{\mathrm{tg} / \mathrm{g}}$ females, yielding control Zeb2 ${ }^{+/ \mathrm{fl}}$, heterozygote $\mathrm{Zeb}^{-/ \mathrm{fl}}$, or $\mathrm{Zeb}^{+/ \Delta \mathrm{Cre}(\mathrm{LacZ}+)}$ and knockout Zeb2 $2^{-/ \Delta \mathrm{Cre}(\mathrm{LacZ}+)}$ embryos. Mice were maintained on a mixed outbred CD1 background. The animal ethics committee of Ghent University approved all experiments performed on mice.

\section{Whole-mount stainings}

Whole-mount immunostaining of the AGM region was performed as described previously. ${ }^{16}$ Primary antibodies used were cKit (2B8; BD Biosciences) and biotinylated CD31 (MEC13.3; BD Biosciences). Secondary antibodies used were goat anti-rat $\operatorname{IgG}$ conjugated to Alexa Fluor 647 (Invitrogen) and Cy3-conjugated streptavidin (Jackson ImmunoResearch Laboratories). For whole-mount staining with 5-bromo-4-chloro-3-indolyl $\beta$-D-galactopyranoside (X-gal), embryos and long bones were dissected in ice-cold PBS, fixed in 4\% paraformaldehyde in PBS at $4^{\circ} \mathrm{C}$ for 1 hour, and washed in PBS. Samples were stained overnight in X-gal staining solution, as described previously. ${ }^{17}$ Sections were counterstained with eosin.

\section{Histology}

Whole embryos or dissected fetal livers and brains were fixed overnight in $4 \%$ paraformaldehyde at $4^{\circ} \mathrm{C}$, processed for paraffin embedding, sectioned $(5 \mu \mathrm{m})$, and stained with hematoxylin and eosin. Tissue sections were double-stained according to the protocol for the Renaissance TSA Biotin System (NEL 700; PerkinElmer) using rabbit-anti-desmin (1:200; Abcam) or rat-anti-mouse CD31 antibody (1:50; BD Biosciences), followed by incubation with either biotinylated anti-rabbit (Vector Laboratories) or biotinylated anti-rat antibody (BD Biosciences), streptavidin-alkaline phosphatase (PerkinElmer), and VECTOR Red Alkaline Substrate (Vector Laboratories). Sections were incubated with rat-anti-mouse CD45 antibody (1:200; BD Pharmingen), followed by the incubation with biotinylated anti-rat antibody and Streptavidin Alexa Fluor 488 (Molecular Probes). For Zeb2 immunohistochemistry, a diaminobenzidine protocol was applied on an automated platform (Discovery XT Ventana; Roche), as described previously. ${ }^{18}$

\section{Flow cytometric analysis}

Cells from E11.5-E12.0 fetal livers or peripheral blood were stained with a mouse lineage cocktail containing cKit and Sca1 antibodies (summarized in supplemental Table 1, available on the Blood Web site; see the Supplemental Materials link at the top of the online article), and analyzed with an LSRII (BD Biosciences) and FACSDiva software (Version 6.1.3) (BD Biosciences). Dead cells were discarded from analysis using SYTOX Blue (1:3000; Molecular Probes). For molecular analysis on HSCs/HPCs, E12.0 fetal liver cells were stained with the antibodies listed in supplemental Table 1. $\mathrm{Lin}^{-} \mathrm{cKit}^{+}$cells were sorted using the Epics Altra FACS (Beckman Coulter). Zeb2 mRNA expression analysis in HSCs/HPCs was performed on FACS-sorted bone marrow subpopulations isolated from adult $\mathrm{C} 57 \mathrm{~B} 1 / 6$ mice. Bone marrow was harvested and subjected to lineage depletion using magnetic beads and columns (Miltenyi Biotec). The $\mathrm{Lin}^{-}$fraction was then stained with the antibodies listed in supplemental Table 1. HSCs $\left(\mathrm{Lin}^{-} \mathrm{cKit}^{+} \mathrm{Sca}{ }^{+} \mathrm{CD} 34\right)$, common myeloid progenitor cells $\left(\mathrm{Lin}^{-} \mathrm{CKit}^{+} \mathrm{Sca} 1^{-} \mathrm{CD} 34^{+} \mathrm{CD} 16 / 32^{\text {low }}\right.$ ), granulocyte/monocyte progenitor cells ( $\mathrm{Lin}^{-} \mathrm{CKit}^{+} \mathrm{Sca}{ }^{-} \mathrm{CD} 34^{+} \mathrm{CD} 16 / 32^{\text {high }}$ ), and megakaryocyte/erythroid progenitor cells $\left(\mathrm{Lin}^{-} \mathrm{cKit}^{+} \mathrm{Sca}{ }^{-} \mathrm{CD} 34^{-} \mathrm{CD} 16 / 32^{\text {low }}\right)$ were sorted using FACSDiva (BD Biosciences).

\section{In vitro hematopoietic progenitor assay}

For definitive hematopoietic colony-forming assays, Methocult GF3434 medium (StemCell Technologies) was used according to the manufacturer's instructions. Colonies were blindly scored after 5-7 days and at 12 days of culture at $37^{\circ} \mathrm{C}$ with $5 \% \mathrm{CO}_{2}$ and $>95 \%$ humidity. Similarly, megakaryocyte differentiation assays were performed using MegaCult-C medium (StemCell Technologies) according to the manufacturer's protocols. Subsequently, cultures were stained with acetylthiocholiniodide for 3 hours. Zeb2-null embryonic stem (ES) cells were generated and provided by D. Huylebroeck's laboratory (L.U. et al, unpublished results). Embryoid body formation was performed following standard procedures and is described in detail in the supplemental Methods.

\section{Transwell migration assay}

Chemotaxis experiments were done in Costar Transwells (6.5-mm diameter, $5-\mu \mathrm{m}$ pore size; Corning). Before migration, fetal liver cells were enriched for cKit expression using anti-mCD117 magnetic beads and columns (Miltenyi Biotec). $4 \times 10^{4}$ (Tie2-Cre) or $1.5 \times 10^{5}$ (Vav-iCre) $\mathrm{cKit}^{+}$cells were seeded in the upper well and for 4 hours allowed to migrate toward no $(0 \mathrm{ng} / \mathrm{mL})$, low $(15 \mathrm{ng} / \mathrm{mL})$, and high $(150 \mathrm{ng} / \mathrm{mL})$ levels of recombinant mSDF- $1 \alpha$ in RPMI medium with $10 \% \mathrm{FCS}$ at $37^{\circ} \mathrm{C}, 5 \% \mathrm{CO}_{2}$, and $>95 \%$ humidity.

\section{Molecular analysis}

Brain samples were dissected, snap-frozen in liquid nitrogen, and processed according to standard protocols. For quantitative RT-PCR (qRT-PCR), RNA was extracted with TRIzol (Invitrogen), treated with DNase I, and purified using an RNeasy Cleanup Mini Column (QIAGEN). cDNA synthesis (Roche or Applied Biosciences) was performed starting from equal amounts of RNA. qRT-PCR was performed on a LightCycler 480 system (Roche) using the SYBR Green I Master Kit (Applied Biosystems). Gene expression was normalized using the housekeeping genes $\beta$-actin, Gapdh, and/or Ywhaz. Primers used in this study are indicated in supplemental Table 2. Technical $(n>3)$ and biological replicates $(n>4$ embryos per genotype) were used in qRT-PCR. Results are expressed as the fold change compared with controls.

Imaging and statistical analyses are described in detail in the supplemental Methods. 


\section{Results}

\section{Zeb2 is expressed in embryonic and adult HSCs}

Zeb2 mRNA expression was previously demonstrated in adult B lymphocytes ${ }^{1}$ and in various embryonic and adult hematopoietic organs. ${ }^{19,20}$ However, these initial studies lacked detailed, cell type-specific marker analysis. To analyze Zeb2 steady-state mRNA expression levels specifically in HSCs and/or lineage-restricted HPCs, qRT-PCR was performed on various FACS-sorted HSC/ HPC populations from adult mouse bone marrow, and compared with the low Zeb2 mRNA levels in normal mouse mammary epithelial cells and the high levels in mouse embryonic fibroblasts. Zeb2-null mouse ES cells were used as a negative control. In all hematopoietic cell samples analyzed, high Zeb2 mRNA levels were detected and were found to be especially high in the bone marrow subsets enriched for early progenitors of various hematopoietic lineages (Figure 1A). The highest expression level of Zeb2 mRNA was detected in the $\mathrm{Lin}^{-} \mathrm{cKit}^{+} \mathrm{Sca}{ }^{+} \mathrm{CD} 34^{-}$cells known to be highly enriched for HSCs, ${ }^{21}$ with 6- to 7-fold higher expression levels of Zeb2 mRNA compared with mouse embryonic fibroblasts.

In the mouse embryo, the first HSCs are generated at E10.5E11.5 in the AGM region within the aorta. Hematopoietic cell clusters are found closely associated with the aortic endothelium, and it is thought that HSC/HPCs arise from the specialized hemogenic endothelium in this vessel. ${ }^{13}$ Using immunohistochemical (IHC) staining analysis, we detected Zeb2 protein in aortic hematopoietic clusters in E10.5 wild-type embryos (Figure 1B). These data showed that Zeb2 mRNA and protein, respectively, was expressed in adult HSCs/HPCs and in aortic hematopoietic clusters from the earliest phases of definitive embryonic hematopoiesis.

\section{Tie2-Cre-mediated loss of Zeb2 results in embryonic lethality at E12.5}

To analyze Zeb2 functions in hematopoiesis, conditional Zeb2knockout mice ${ }^{14}$ were crossed with a Tie2-Cre line, ${ }^{22}$ producing Cre recombinase in endothelial cells and their progeny, including HSCs and all definitive blood cells. ${ }^{23}$ Loss of Zeb2 resulted in embryonic lethality around E12.5-E13.5 (supplemental Table 3). No obvious defects were observed before E11.5, suggesting that the first wave of primitive yolk sac hematopoiesis is not drastically

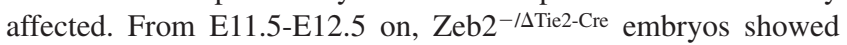
intracerebral hemorrhages and pooling of primitive nucleated erythrocytes in the head and neural tube at E12.5 (Figure 2A). Histologic analysis of the mutant embryos showed hemorrhages specifically in neural tissues and predominantly in developing ganglia and forebrain ganglionic eminences (Figure 2B). Dissected E12.5 mutant fetal livers showed signs of anemia (data not shown). In addition, the mutant embryos showed variable heart malformations, including pericardial bleeding, ventricular wall thinning, and dilatation of cardinal veins (supplemental Figure 1A). Because vascular patterning defects may cause the observed cephalic bleeding, the mice were crossed with R26-LacZ reporter transgenic mice, ${ }^{24}$ and whole-mount straining with $\mathrm{X}$-gal was performed on E11.5-E12.5 embryos. No drastic changes in vasculature patterning were observed between knockout Zeb2 $2^{-/ \Delta T i e 2-C r e}$; R26-LacZ ${ }^{+/ \Delta T i e 2-C r e}$ and the heterozygote Zeb2 ${ }^{+/ \Delta \text { Tie2-Cre; }}$

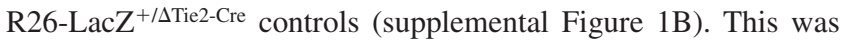
confirmed by whole-mount isolectin-B4 stainings of E12.0 hindbrains (supplemental Figure 1C) and CD31 IHC on E11.5-E12.5 whole-embryo sections (supplemental Figure 1D).
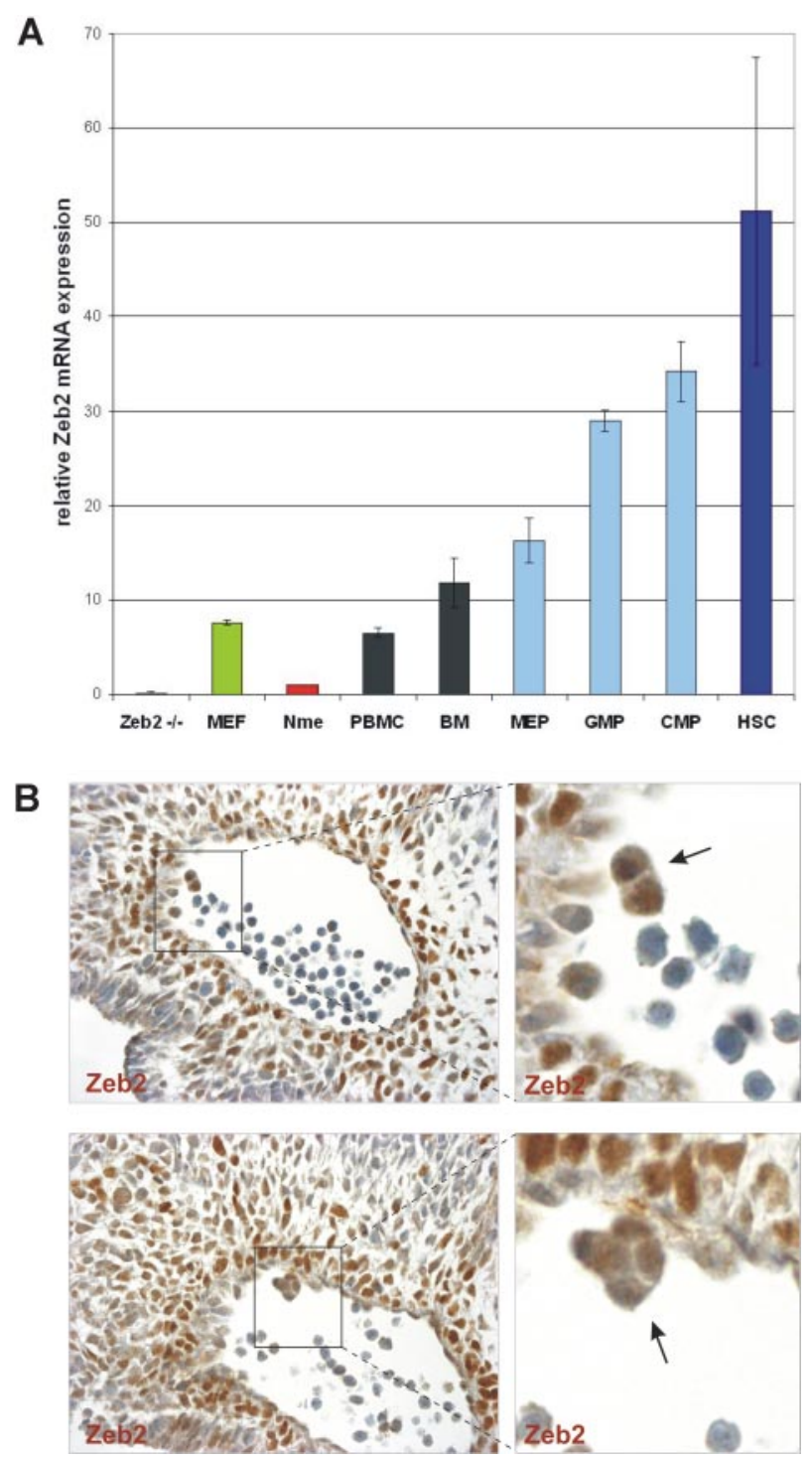

Figure 1. Zeb2 is highly expressed in embryonic hematopoietic clusters and adult HSCs. (A) qRT-PCR using Zeb2-specific primers on total RNA samples prepared from peripheral blood mononuclear cells (PBMC), bone marrow (BM), FACS-sorted hematopoietic progenitor cells (MEP, megakaryocyte/erythroid progenitors; GMP, granulocyte/monocyte progenitors; CMP, common myeloid progenitors), and HSCs from adult mice. As a negative control, RNA from feeder-free Zeb2-null ES cells and normal mammary epithelial (Nme) mouse cells were used. As a positive control for Zeb2 mRNA expression, RNA from mouse embryonic fibroblasts (MEF) was used. Bars represent mean \pm SD. Two independent sorting experiments were performed and qRT-PCR was done in triplicate on biological duplicates (B) Zeb2 immunohistochemical staining of sections of the AGM region of a E10.5 embryo (400× magnification), demonstrating Zeb2 protein presence in hematopoietic clusters (arrows) budding from the hemogenic endothelium of the dorsal aorta.

\section{Zeb2 is not essential for HSC formation}

The phenotype observed with Tie2-Cre-mediated Zeb2 deletion (normal yolk sac hematopoiesis, hemorrhaging, cardiovascular defects, E12.5 lethality) is reminiscent of the phenotypes associated with ubiquitous loss of the hematopoietic transcriptional regulators AML/Runx1, ${ }^{25}$ Fli1, ${ }^{26}$ and Gata3. ${ }^{27}$ Runx1-knockout embryos are deficient in AGM HSCs ${ }^{16}$ and lack intraarterial hematopoietic clusters, suggesting that Zeb2 deletion may also affect hematopoietic cluster formation. To examine this, wholemount CD31 (endothelial)/cKit (HSC) double staining of E11.5 
From bloodjournal.hematologylibrary.org at KU Leuven on September 25, 2012. For personal use only.

A
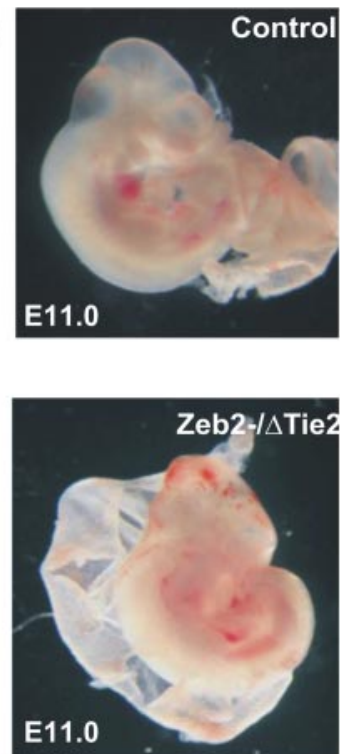
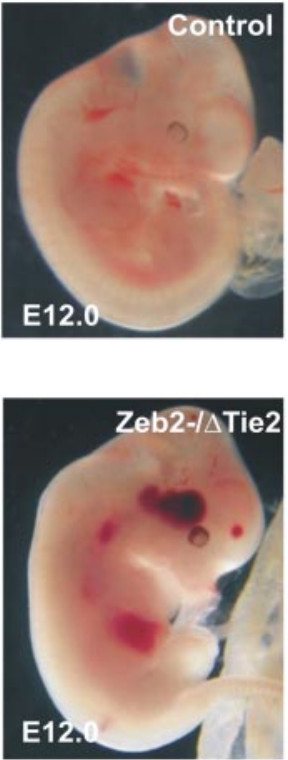
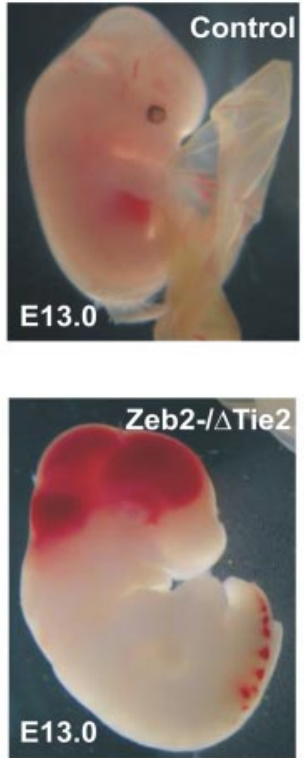

B
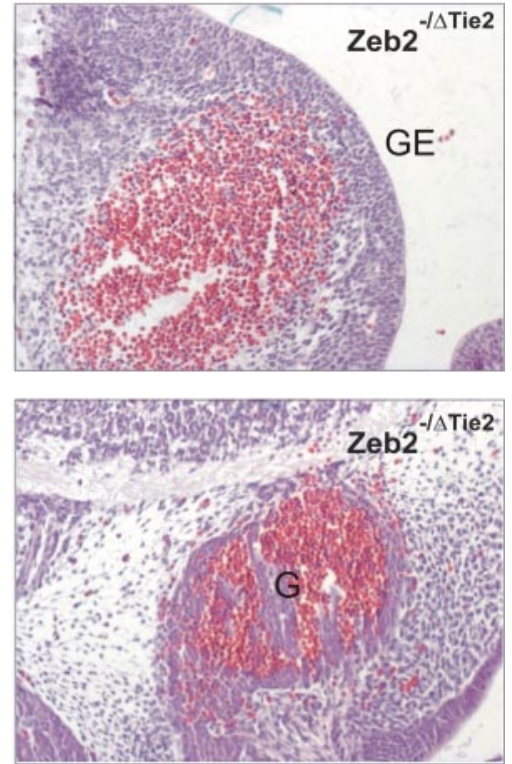

Figure 2. Tie2-cre-mediated loss of Zeb2 causes intracranial hemorrhaging at E12.5. (A) Zeb2-/ATie2-Cre hemorrhagic phenotype. (B) Hematoxylin/eosin staining of E12.5 Zeb2 $2^{-/ \Delta T i e 2-C r e}$ embryo (200X magnification) showing bleeding especially in the germinal layer and ganglia/ganglionic eminences of the developing brain. GE indicates ganglionic eminence; and $\mathrm{G}$, ganglion.

AGM were performed. No significant differences in aortic hemato-

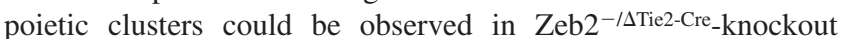
AGM regions compared with littermate controls (Figure 3A). Intercrossings with R26-LacZ reporter mice followed by wholemount staining with X-gal confirmed Tie2-mediated Cre recombination in these aortic hematopoietic clusters (Figure 3B). Efficient Zeb2 deletion was also documented by the absence of Zeb2 protein in the hematopoietic clusters (Figure 3C). These results suggest that Zeb2 is not essential for the generation of hematopoietic clusters, but may be required for later HPC/ HSC function.

\section{Vav-iCre-mediated Zeb2 deletion recapitulates the Tie2-Cre Zeb2-knockout phenotype}

To further examine the role of Zeb2 in hematopoiesis and rule out the potential contribution of loss of endothelial Zeb2 expression to the hemorrhagic phenotype, Zeb2 conditional-knockout mice were
Figure 3. Zeb2 is not essential for the formation of hematopoietic clusters in the AGM. (A) Quantification (right panel) of intraaortic hematopoietic clusters budding from the hemogenic endothelium by wholemount CD31/cKit immunofluorescence staining of AGM region of E11.5 control wild-type and Zeb2- $/ \Delta$ Tie2-Cre embryos (400× magnification) Bars represent mean \pm SD. (B) Hematoxylin/eosin staining (200× magnification on the left, $600 \times$ magnification on the right), X-gal staining for LacZ expression showing Cre excision in budding hematopoietic clusters from excised ROSA26-LacZ allele (arrows in B) and (C) IHC with a Zeb2 antibody on AGM sections of E10.5 Zeb2-/ATie2-Cre embryo (200X magnification on the left, $600 \times$ magnification on the right) showing lack of Zeb2 immunoreactivity in the budding hematopoietic clusters (arrows). (D) Vav-iCremediated loss of Zeb2 recapitulates the Zeb2- ${ }^{-\Delta \text { Tie2-Cre }}$ cephalic bleeding phenotype. Zeb2-/AVav-iCre mice die around birth and show massive cerebral bleeding (arrow).
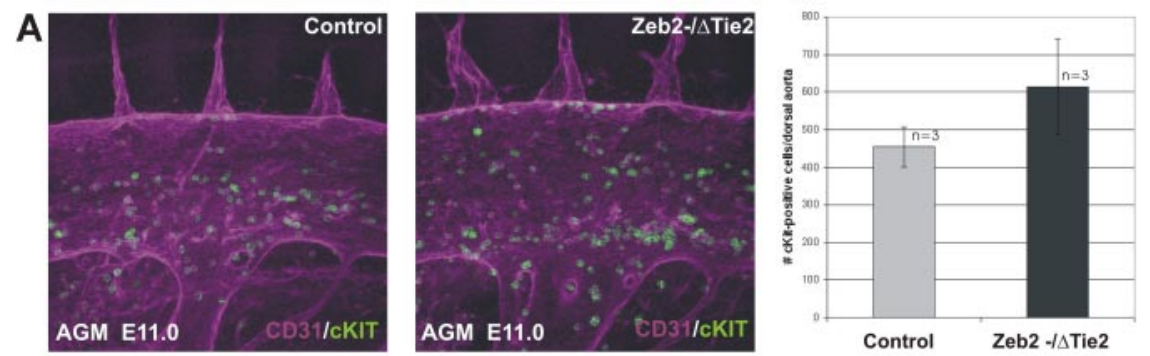

B
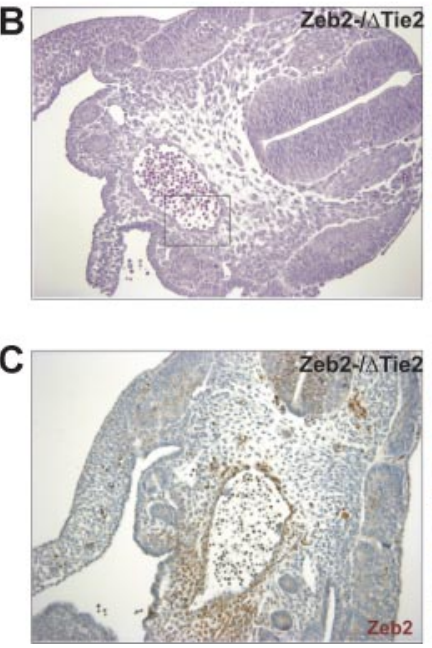
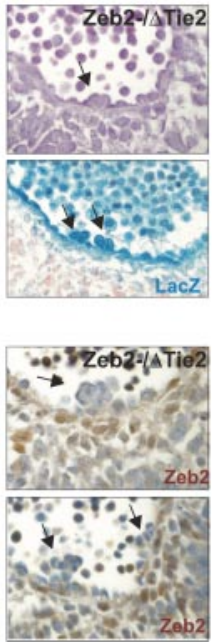

D

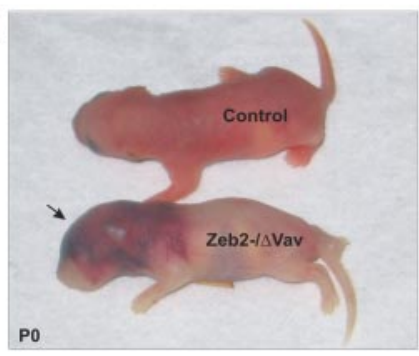


crossed with a Vav-iCre line ${ }^{28}$ that produces the Cre recombinase specifically in hematopoietic cells (supplemental Figure 2A-B), but only after HSC formation. ${ }^{29}$ Furthermore, the comparison of the phenotypes associated with Tie2-Cre- and Vav-iCre-mediated loss of Zeb2 allowed us to investigate at what stage in development the expression of Zeb2 is essential for hematopoiesis: before or after HSC formation. ${ }^{16}$ In the Zeb2 ${ }^{-/ \Delta V a v-i C r e}$ embryos, a phenotype very similar to that seen in the Zeb2 $2^{-/ \Delta T i e 2-C r e}$ knockouts could be

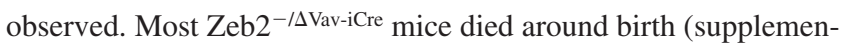
tal Table 4) and showed massive intracranial bleeding similar to the

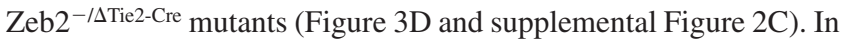
addition, peripheral blood counts of surviving E18.0 Zeb ${ }^{-/ \Delta V a v-i C r e}$ embryos showed significantly decreased numbers of RBCs and thrombocytes and an increase in nucleated erythroid progenitors (supplemental Table 5 and supplemental Figure 2D).

\section{Zeb2 is essential for definitive embryonic HSC differentiation}

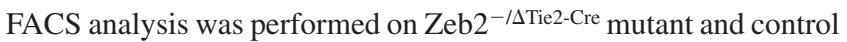
E12.0 fetal livers to detect and quantify the number of phenotypic HSCs $\left(\mathrm{Lin}^{-} \mathrm{Sca}^{+}{ }^{+} \mathrm{Kit}^{+}\right.$, referred to as LSK cells) per fetal liver. LSK cells were present in the fetal livers of Zeb2 mutant embryos (Figure 4A), implying that Zeb2-deficient HSCs are not impaired in reaching the fetal liver. We observed a significant 2- to 3-fold increase in LSK cells, whereas more committed early progenitors $\left(\mathrm{Lin}^{-} \mathrm{Scal}^{-} \mathrm{cKit}^{+}\right)$were decreased by $40 \%$ in the fetal livers of Zeb2 $2^{-/ \Delta T i e 2-C r e}$ embryos, suggesting a defect in HSC differentiation (Figure 4A and supplemental Table 6).

To further investigate the role of Zeb2 in HSC differentiation, CFU assays in methylcellulose with cells isolated from various hematopoietic organs from Zeb2-/ATie2-Cre E10.5 yolk sacs, E11.5 AGM regions, E11.5-E12.0 fetal livers, and E12.0 peripheral blood were analyzed and all showed a dramatic impairment in hematopoietic colony formation compared with the controls (Figure 4B). These results indicate a severe block in hematopoietic differentiation in all lineages (Figure 4C). A significant, albeit less severe

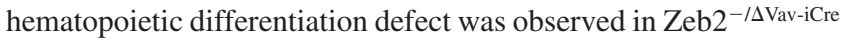
fetal livers (Figure 4D). Consistent with these findings, Zeb2-null ES cells were also unable to undergo hematopoietic differentiation (Figure 4E). Zeb2-null ES cells showed no differentiation defect in other mesodermally derived cell types in these cultures, including beating cardiac muscle cells and $\mathrm{CD} 31^{+}$endothelial cells (data not shown). These data demonstrate that Zeb2 is essential for definitive hematopoietic differentiation at an early stage in definitive hematopoiesis. To provide molecular insight into this differentiation block, $\mathrm{Lin}^{-} \mathrm{CKit}^{+}$progenitor cells were sorted from fetal livers, and the expression of several important hematopoietic transcription factors was examined by qRT-PCR. Mild but significant increases in Runx1 mRNA expression levels were observed, but no other significant alterations for Fli1, Gata1/2, Tal1, or Lmo2 were found (Figure 4F).

\section{Loss of Zeb2 alters hematopoietic progenitor localization in the fetal liver}

Further analysis of the mutant fetal liver architecture suggested subtle developmental changes and prompted us to carry out a more detailed IHC and molecular marker analysis in both conditional mouse models. Again, no obvious alterations were seen in the vascular patterning of the developing livers (data not shown), but significant alterations could be observed in the localization of the HPCs (using the pan-hematopoietic marker CD45). In the
Zeb2-/ATie2-Cre mutant fetal livers, an increased number of CD45 ${ }^{+}$ cells were seen clustering in or close to the major vessels, whereas the overall pattern was punctate in control fetal livers (Figure

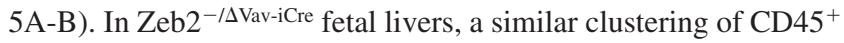
cells was observed (Figure 5C), but not in the immediate vicinity of the vessels. In addition, homing of hematopoietic cells to the bone

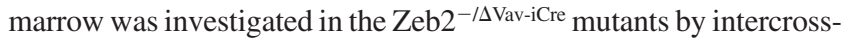
ing with the R26-LacZ reporter mice and whole-mount staining with X-gal. Clear decreases in targeted hematopoietic cells (X-gal ${ }^{+}$ cells) were seen in the Zeb2 mutant bone marrow compared with controls (Figure 5D). Flow cytometric analysis on peripheral blood samples revealed a significant reduction of circulating HSC/HPC cells in Zeb2 mutant embryos (Figure 5E). These data suggest increased adhesive properties of HSC/HPCs in the fetal liver and reduced mobilization and colonization of the bone marrow cavity.

Because Zeb2 has previously been demonstrated to repress various genes involved in cell-cell adhesion and cell-matrix interactions, ${ }^{3}$ we analyzed by qRT-PCR the mRNA levels of a selected panel of candidate Zeb2 target genes and/or adhesion genes previously demonstrated to be essential for HSC adhesion

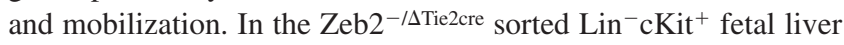
cells, a significant, 2.7-fold increase of integrin $\beta 1$ (Itgb1) and a 15.5-fold increase of Cxcr4 mRNA was observed (Figure 5F). No such changes were found for integrin $\alpha 4$ (Itga4), integrin $\alpha 5$ (Itga5), integrin $\alpha 6$ (Itga6), integrin $\beta 3$ (Itgb3), E-cadherin (Cdh1), or N-cadherin (Cdh2) (data not shown).

Subsequently, the relevance of this increased Cxcr4 expression was tested with a Transwell migration assay toward low $(15 \mathrm{ng} / \mathrm{mL})$ and high $(150 \mathrm{ng} / \mathrm{mL})$ concentrations of the cognate ligand Sdf- $1 \alpha$. Compared with their littermate controls, $\mathrm{cKit}^{+}$-enriched fetal liver

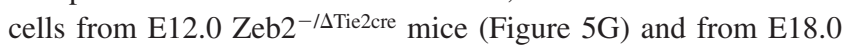
Zeb2 ${ }^{-/ \Delta \text { Vav-iCre }}$ mice (Figure $5 \mathrm{H}$ ) showed a significant 2- to 3-fold increased migration, especially toward the lower Sdf- $1 \alpha$ concentration.

\section{Hematopoietic-specific loss of Zeb2 disrupts Ang1/Tie2 signaling and affects pericyte coverage of the vasculature in the developing brain}

We next investigated whether the retention of HSCs/HPCs in the fetal liver and the decrease in circulating HPCs may underlie the embryonic lethality and intracephalic bleeding. Circulating HSCs/ HPCs during this stage of embryonic development have been shown to be the source of important angiogenic growth factors, including angiopoietin-1 (Ang1), ${ }^{30,31}$ which is essential for the pericyte recruitment that provides the necessary vessel stability to prevent vascular leakage. Loss of HSC formation in Runx1deficient embryos, and therefore the loss of these crucial angiogenic factors, was postulated to be the cause of the observed hemorrhagic lethality. ${ }^{30}$ qRT-PCR analysis showed significant decreases in Ang1 mRNA expression levels in the heads of Zeb2-knockout embryos compared with controls (Figure 6A). In addition, double staining of desmin (pericytes)/CD31(endothelial cells) on sections of E15.5-E17.5 Zeb2 ${ }^{-/ \Delta V a v-i C r e}$ cephalic regions revealed drastic decreases in pericyte coverage of the vessels in the mutant brains (Figure 6B). These data strongly suggest that the underlying cause of the vascular hemorrhages observed after hematopoietic-specific loss of Zeb2 was due to altered pericyte coverage of the cephalic vasculature that was associated with decreased Ang1 levels and causally linked to a lack of circulating HSCs/HPCs. 

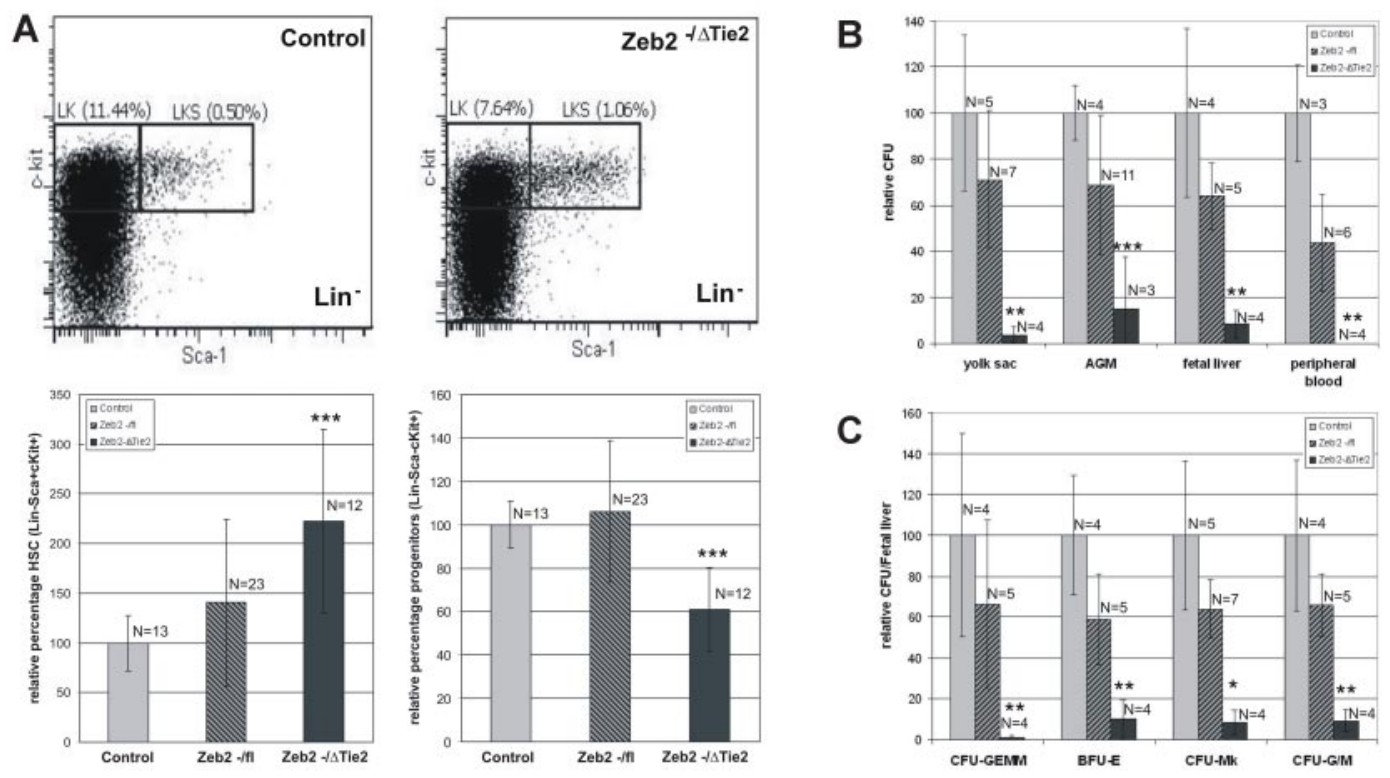

\section{C}

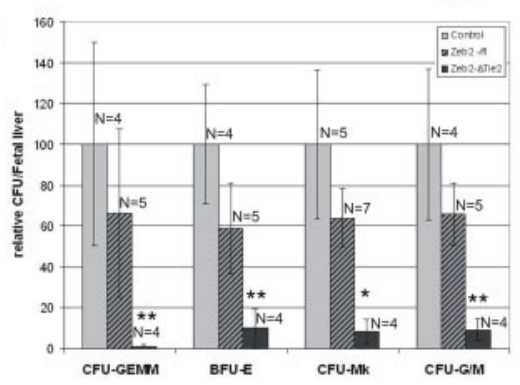

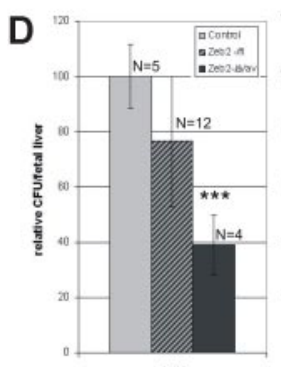

CFU
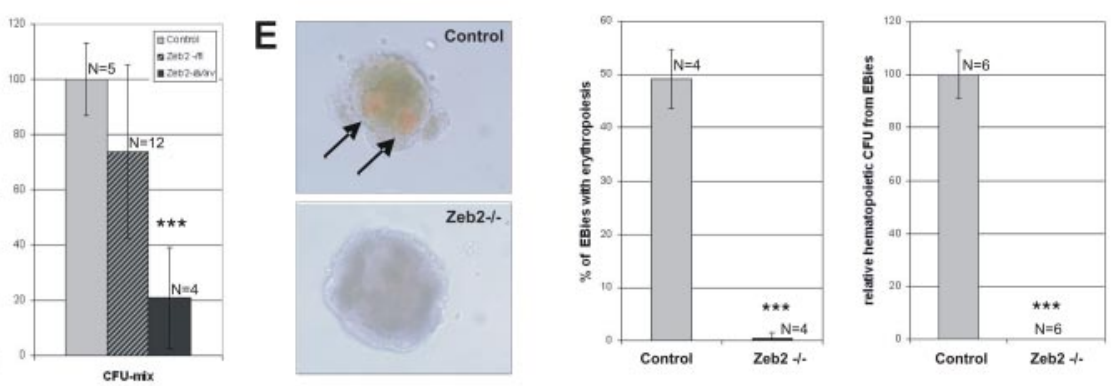

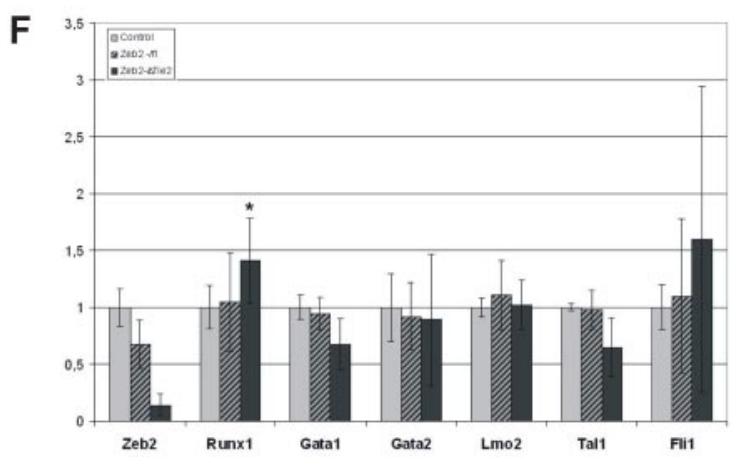

Figure 4. Zeb2 is not essential for LSK marker-enriched HSC migration to the fetal liver, but blocks definitive hematopoietic differentiation at an early stage. (A) FACS quantification of HSCs (LSK) and early progenitors (LK, Lin ${ }^{-}$cKit $^{+}$Sca1 ${ }^{-}$) in wild-type and mutant fetal liver. Relative 2.2 -fold increase of HSCs (LSK) and relative $40 \%$ decrease of early committed progenitors (LK) in Zeb2-/Tie2-Cre fetal livers compared with wild-type littermates. (B) Quantification of total hematopoietic colonies formed after in vitro differentiation in methylcellulose of cells isolated from various hematopoietic organs: E10.5 yolk sacs, E11.5 AGM, E11.5-E12.0 fetal livers, and E12.0 peripheral blood. Equal amounts of cells per organ were plated in triplicate, and the average number of colonies obtained was normalized to the wild-type controls. For each organ, at least 2 experiments were performed. (C) Quantification of mixed (CFU-GEMM), erythroid (BFU-E), megakaryocyte (CFU-Mk), and granulocyte/monocyte (CFU-G/M) colonies formed after in vitro differentiation of E11.5 fetal livers cells of Zeb2 $-\Delta$ Tie2-Cre and heterozygote embryos relative to control littermates. (D) Quantification of total (CFU) and mixed (CFU-GEMM) hematopoietic colonies formed after in vitro differentiation of cells isolated from E14.5 fetal livers of Zeb2 ${ }^{-/ \Delta V a v-i C r e}$ and heterozygote embryos relative to control littermates. (E) In vitro differentiation of wild-type and Zeb2-null ES cells and quantification of hemoglobin-positive (arrows) embryoid bodies at day 9. Shown is the quantification of hematopoietic colonies formed after in vitro differentiation of Zeb2-null embryoid body-derived cells relative to wild-type embryoid body-derived cells. (F) qRT-PCR on total RNA from sorted Lin ${ }^{-} \mathrm{CKit}^{+} \mathrm{HSC}$ /HPCs from mutant and control E12.0 fetal livers, analyzing the mRNA levels of various important hematopoietic transcription factors involved in HSC differentiation. Two independent sorting experiments were performed, and qRT-PCR was done in triplicate on biological replicates ( $\mathrm{n}>4$ per genotype). Bars in A-F represent mean $\pm \mathrm{SD} ;{ }^{*} P<.05 ;{ }^{\star \star} P<.01 ;{ }^{* \star \star} P<.001$.

\section{Discussion}

In several recent studies, it was shown that up-regulated expression of EMT modulators stimulates epithelial cells to adopt stem cell characteristics in vitro ${ }^{7}$ and CSC characteristics in vivo. ${ }^{9}$ We have documented high Zeb2 mRNA levels in adult HSCs/HPCs and protein expression in budding hematopoietic clusters from the dorsal aorta at E10.5 that are the original sites of HSC/HPC development. ${ }^{12}$ To investigate whether Zeb2 could also be involved 
From bloodjournal.hematologylibrary.org at KU Leuven on September 25, 2012. For personal use only.

A
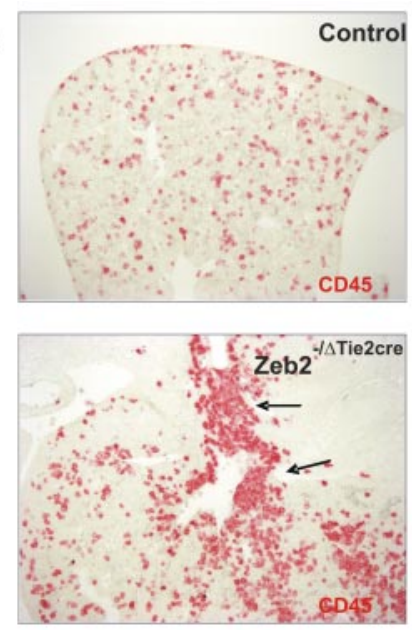

C
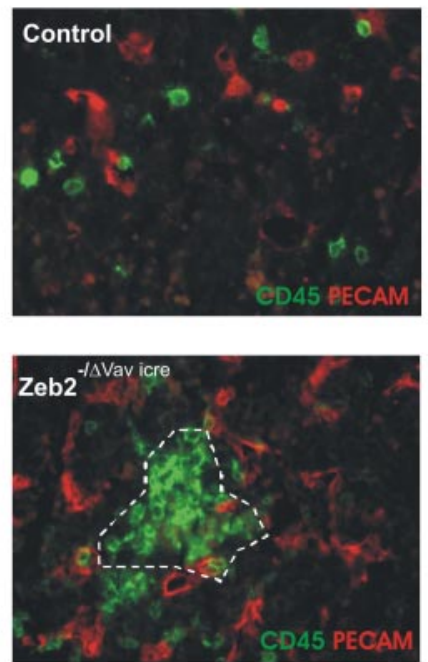
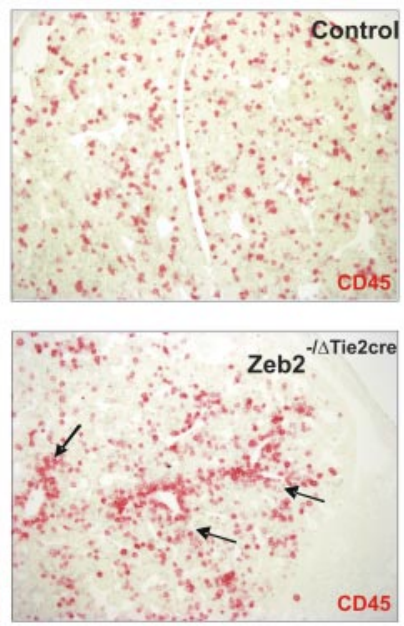

B
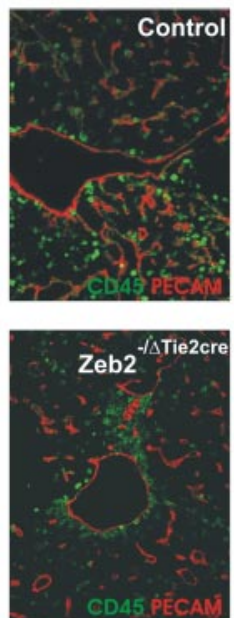
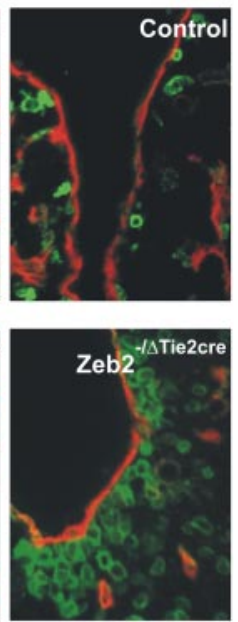

D
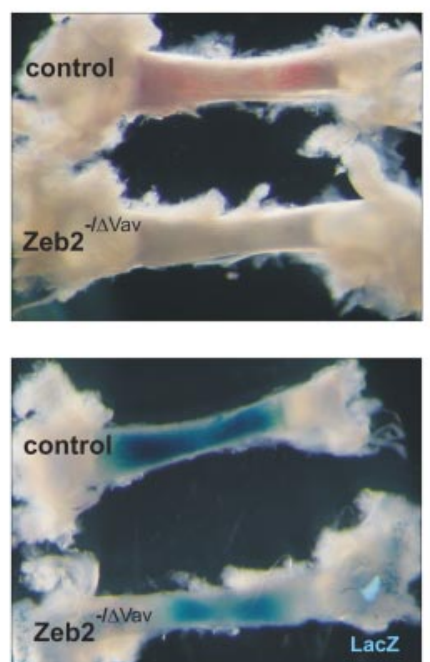
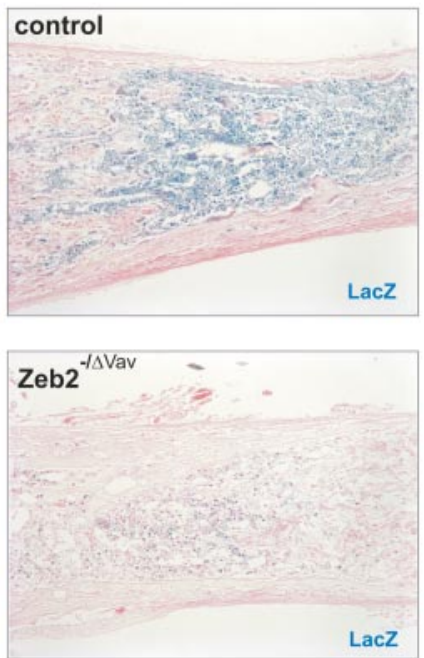

E

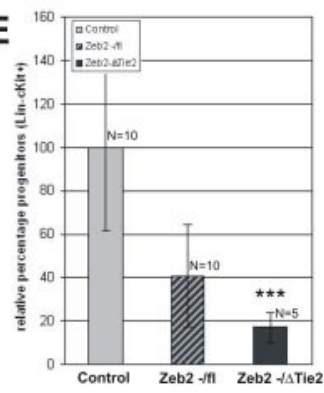

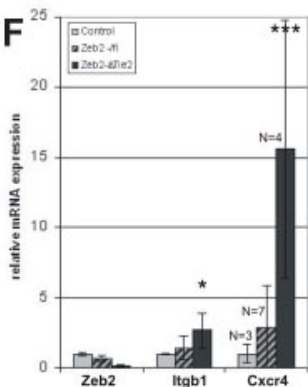
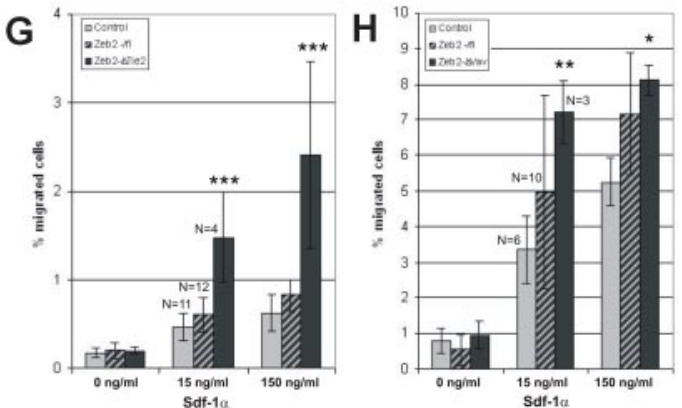

Figure 5. Loss of Zeb2 alters adhesive properties and localization of hematopoietic progenitors in the fetal liver, ablating their mobilization/homing to the bone marrow. (A) Localization of hematopoietic cells in E11.5 wild-type and Zeb2-/ATie2-Cre fetal livers using the pan-hematopoietic marker CD45 (100 $\times$ magnification) showing

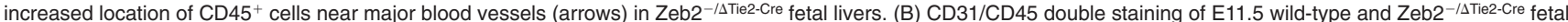
livers (100× and $400 \times$ magnification), confirming increased localization of CD45+ cells near CD31+ vasculature (arrows). (C) CD45/CD31 double-immunofluorescence staining (400 $\times$ magnification) on E16.5 wild-type and Zeb2 ${ }^{-/ \Delta V a v-i C r e}$ fetal livers showing clustering within the fetal liver at a distance from the CD31+ vasculature (dotted line). (D) Decreased mobilization of hematopoietic cells to bone marrow could be observed in Zeb2-/AVav-icre neonates by analyzing the redness (from hemoglobinized RBCs) and whole-mount X-gal staining of tibias (left panels). Eosin-counterstained histologic sections of these bones (100 $\times$ magnification) showing decreased numbers of X-Gal-stained Cre-excised hematopoietic cells in Zeb2 ${ }^{-/ \Delta V a v-i C r e}$ tibias. (E) FACS quantification of hematopoietic progenitors (Lin ${ }^{-} \mathrm{cKit}^{+}$) in the peripheral blood of E12.0 Zeb2 ${ }^{-/ \Delta T i e 2-C r e}$ and heterozygote embryos relative to the wild-type control littermates. (F) qRT-PCR on total mRNA analyzing the expression levels of integrin $\beta 1$ and Cxcr4 involved in HSC mobilization from sorted HSCs/HPCs (Lin ${ }^{-}$cKit) from mutant Zeb2 ${ }^{-/ \Delta T i e 2-C r e ~ a n d ~ c o n t r o l ~ E 12.0 ~ f e t a l ~ l i v e r s . ~ T w o ~ i n d e p e n d e n t ~ s o r t i n g ~ e x p e r i m e n t s ~ w e r e ~ p e r f o r m e d ~ a n d ~ q R T-P C R ~}$ was done in triplicate on biological replicates ( $n>4$ per genotype). (G) Zeb2 ${ }^{-/ \Delta T i e 2-C r e}$ and $(H) Z_{\text {Zeb2 }}{ }^{-/ \Delta V a v-i C r e}$ cKit $^{+}$-enriched fetal liver cells show significant increased migration potential toward low $(15 \mathrm{ng} / \mathrm{mL})$ and high $(150 \mathrm{ng} / \mathrm{mL})$ recombinant $\mathrm{mSDF}-1$ a concentrations in an in vitro Transwell migration assay compared with cKit ${ }^{+}$-enriched fetal liver cells from control littermates. Bars in panels $\mathrm{E}$ through $\mathrm{H}$ represent mean $\pm \mathrm{SD} ;{ }^{\star} P<.05 ;{ }^{\star \star} P<.01 ;{ }^{\star \star \star} P<.001$.

in the physiologic formation of stem cells in vivo beyond CSCs, we studied its role in the definitive stem cells of the hematopoietic system. We did this through conditional deletion of Zeb2 at different stages of early mouse hematopoiesis using 2 independent Cre-based approaches.

In wild-type mice, the first definitive adult-type HSCs originate 

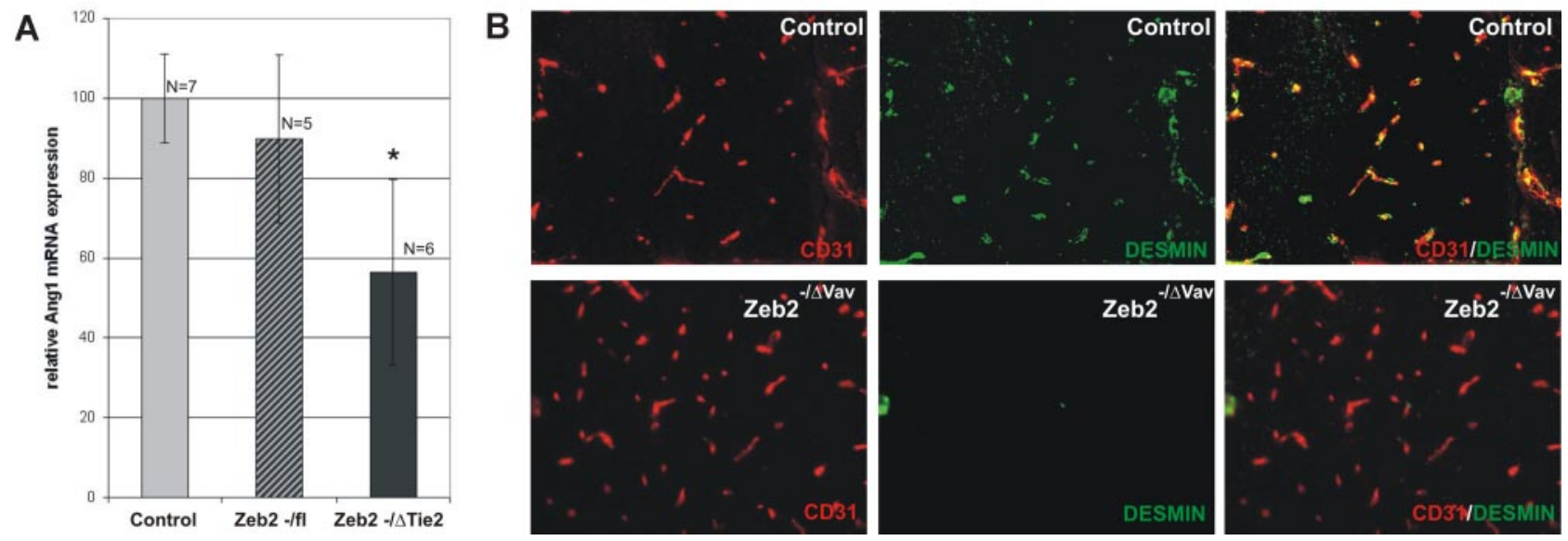

Figure 6. Hematopoietic-specific loss of Zeb2 decreases Ang1 expression in the head and is associated with decreased desmin ${ }^{+}$pericyte coverage of the head vasculature. (A) qRT-PCR for angiopoietin-1 on RNA isolated from wild-type and Zeb2-/ATie2-Cre heads. Technical $(n>3)$ and biological replicates ( $\mathrm{n}>5$ embryos per genotype) were used. Bars represent mean $\pm \mathrm{SD} ;{ }^{\star} P<.05 ;{ }^{\star \star} P<.01 ;{ }^{\star \star \star} P<.001$. (B) CD31/desmin immunofluorescence staining on Zeb2 ${ }^{-/ \Delta V a v-i C r e}$ and control wild-type head vasculature showing lack of desmin ${ }^{+}$cell coverage of the head vasculature in Zeb2 ${ }^{-/ \Delta \text { Vav-iCre }}$ neonates.

in the aorta at E10.5-E11.5 12 and are generated as part of the hematopoietic clusters that bud out in an EMT-like process from hemogenic endothelial cells within the ventral wall of the dorsal aorta. ${ }^{13}$ Through the conditional deletion of Zeb2 in the hemogenic endothelium (using the Tie2-Cre transgenic line ${ }^{22}$ along with whole-mount quantification of $\mathrm{cKit}^{+}$hematopoietic clusters), we could address the putative role of Zeb2 in hematopoietic cluster

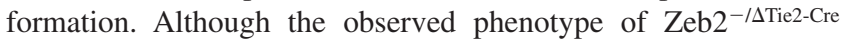
embryos was very similar to the gross phenotype previously reported after the deletion of several genes essential for early definitive hematopoiesis at the AGM region (with Runxl being very well characterized ${ }^{25}$ ), no differences were seen in the number of budding hematopoietic clusters, whereas in Runx1-deficient embryos no clusters form at all. ${ }^{16}$ In contrast, a significant 2- to 3 -fold increase of LSK cells was detected in mutant fetal livers, which might have been caused by the increased formation of HSCs in the AGM, because we could document a trend toward a modest

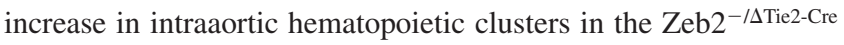
mutants compared with control AGMs. This increase in LSK cells in the Zeb2 $2^{-/ \Delta T i e 2-C r e}$ fetal livers may be indicative of a compensatory feedback loop to overcome the hematopoietic differentiation block and/or the retention of the hematopoietic progenitors in the fetal liver. In addition, this increase in LSK numbers could reflect the mild but significant increases in Runx 1 expression observed in FACS-sorted Zeb2 ${ }^{-/ \Delta \mathrm{Tie} 2-\mathrm{Cre}} \mathrm{Lin}^{-} \mathrm{cKit}^{+}$progenitor cells.

Using the Vav-iCre line, which is only active after HSC formation and not in the vascular endothelium, we could recapitulate several aspects of the phenotype seen using the Tie2-Cre approach. This confirms that Zeb2 plays key roles in definitive hematopoiesis after HSC formation. However, the embryonic lethality in the Vav-iCre approach occurs later and the arrest in hematopoietic differentiation is less profound. This is most likely because of the timing and/or lower efficiency of the Cre recombination associated with this Cre line, as has been reported previously. ${ }^{29}$ These in vivo data suggest that Zeb2 is not directly involved in the formation of hematopoietic clusters in the AGM but instead controls HSC/HPC differentiation potential and $\mathrm{Lin}^{-} \mathrm{cKit}^{+}$progenitor mobilization (Figure 7 is a hypothetical model).

The recapitulation of important Tie2-Cre phenotypes using the hematopoietic-specific Vav-iCre transgenic line implies a hematopoietic origin for the hematopoietic differentiation block and for the cephalic bleeding associated with the aberrant clustering of hematopoietic progenitors in the fetal liver. However, it does not exclude the potential involvement of other cell types through paracrine signaling pathways. Indeed, this has been observed in neurogenesis and gliogenesis in the embryonic brain cortex, ${ }^{18}$ where Zeb2 in postmitotic cells regulates the expression of $N t-3$ and $F g f-9$ that act on the precursor cells, all of which are Zeb2 negative. Furthermore, in the ubiquitous Zeb2 knockout, ${ }^{15}$ Krox20 mRNA expression is lost from rhombomeres 3 and 5, whereas Zeb2 is only present in the flanking rhombomeres of the hindbrain, suggesting again a non-cell-autonomous action of Zeb2. In our mouse model, we propose that the vessel hemorrhagic phenotype results from the ablation of essential angiogenic factors, including Ang1, which is expressed by circulating HSCs/HPCs, influencing vessel stability via indirect effects on both endothelial cells and pericytes (Figure 7).

The hematopoietic differentiation defect was not only seen in vitro in ES-cell differentiation assays, but also in methylcellulose cultures from various organs (yolk sac, AGM, fetal live, and peripheral blood), and finally in vivo by showing a reduction of mature hematopoietic cells in the mutants. Therefore, we do not expect that the block in differentiation is overtly due to environmental defects, and therefore we suggest that we have revealed a cell-autonomous role for Zeb2 in hematopoietic differentiation. However, to confirm the stem-cell-autonomous nature of the hematopoietic differentiation block, an ideal future experiment would be to analyze the persistence of the phenotype after transplantation of mutant cells in a competitive transplantation setting. Nonetheless, loss of Zeb2 did not result in dramatically altered mRNA expression levels of various important hematopoietic transcription factors previously implicated in controlling hematopoietic cell differentiation. This does not rule out the idea that the minor but significant increases in Runx 1 may contribute to the observed hematopoietic block. In addition, Zeb2 may affect directly or indirectly (via other Zeb2-interacting effectors or transcription cofactors such as activated Smads, ${ }^{2}$ the NuRD chromatin remodeling/corepressor complex, ${ }^{32}$ or $\mathrm{CtBP}^{1}$ ) their transcriptional activity and functionality.

Aberrant localization of HSCs/HPCs could be observed in both the Zeb2 ${ }^{-/ \Delta \text { Tie2-Cre }}$ and Zeb2 $2^{-/ \Delta V a v-i C r e}$ fetal livers, and the decreased homing (in Zeb2 ${ }^{-/ \Delta V a v-i C r e}$ mutants) to the bone marrow can most probably be explained by altered adhesive capacities and migration 
A Wild type

FETAL LIVER
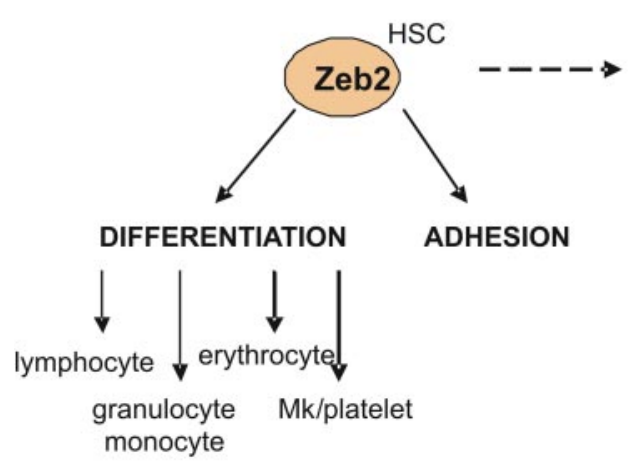

\section{B Zeb2 knockout}

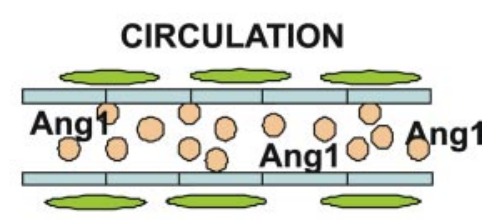

(1)
FETAL LIVER

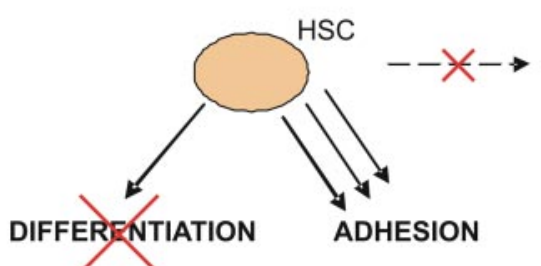

Cxcr4
integrin $\beta 1 \uparrow$ potential of HSCs/HPCs. The increased clustering of hematopoietic cells using the Tie2-Cre approach is slightly different from the results obtained with the Vav-iCre line. This can again be explained

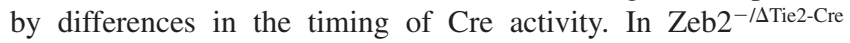
embryos, HSCs/HPCs and their progeny lose their Zeb2 expression levels before homing into the fetal liver, and it appears that they cannot properly invade fetal livers as they cluster near the major vessels as soon as they enter the tissue via the circulation. In the Vav-iCre approach, Cre-mediated recombination only takes place in cells that have already homed to the fetal liver, ${ }^{16}$ and these cells seem to have problems leaving the fetal liver. Zeb2 directly down-regulates the transcription of a large number of genes encoding for essential cell-cell and cell-matrix adhesion proteins, including cadherins ${ }^{7}$ and integrins. ${ }^{1}$ We found in $\mathrm{Lin}^{-} \mathrm{CKit}^{+}$ HSCs/HPCs sorted from E12.0 Zeb2-/ATie2cre fetal livers a significant increase in $\beta 1$ integrin and Cxcr4 mRNA levels. It was demonstrated previously that $\beta 1$ integrin is crucial for HSC adhesion and homing to the fetal liver ${ }^{33}$ and bone marrow during embryonic development and in adult mice. ${ }^{34}$ Either functional blocking or gene deletion of $\beta 1$ integrin resulted in the accumulation of HSCs/HPCs in the peripheral blood. ${ }^{34}$ In addition, the G-protein-coupled chemokine receptor $\mathrm{Cxcr} 4$ has been shown to play a pivotal role in stem cell mobilization. ${ }^{35}$ Cxcr4 is a 7-span transmembrane receptor for stromal derived factor-1a (Sdf-1 $\alpha$ ). Sdf- $1 \alpha$ is highly expressed in the fetal liver endothelium and bone marrow stroma and acts as a strong chemoattractant for HSCs/ HPCs. ${ }^{36}$ Sdf- $1 \alpha$ induces an integrin-mediated migration arrest of hematopoietic progenitors and is essential for the homing and retention of progenitors into the bone marrow. ${ }^{37}$ Raising the plasma levels of Sdf- $1 \alpha$ by intravenous injection of Sdf- $1 \alpha$-expressing adenovirus $^{38}$ or inhibition of $\mathrm{Cxcr}^{39}{ }^{39}$ leads to mobilization of HSCs/HPCs. No previous in vivo Cxcr4 overexpression experiments have been reported at the fetal liver stage, and no data exist
Figure 7. Working model on the role of Zeb2 in HSC/HPC biology. (A) Top panel depicts the wild-type situation in which Zeb2 expression in the HSCs/HPCs of the fetal liver simultaneously regulates their differentiation potential and plays a key role in their proper adhesion/ localization and mobility. Zeb2 expression in HSCs/HPCs allows them to enter the circulation and provide the necessary angiogenic factors, including Ang1, essential for pericyte recruitment and vessel stability. (B) Bottom panel depicts the situation on hematopoietic-specific loss of Zeb2 expression. Zeb2-null fetal liver HSCs/HPCs are blocked in their differentiation potential and show increased adhesion that is correlated with increased Cxcr4 and integrin $\beta 1$ expression. This results in immobility/ retention of HSCs/HPCs in the fetal liver, preventing them from reentering the circulation, which subsequently results in a systemic decrease of essential angiogenic factors, including Ang1, and a loss of pericyte coverage of the head vessels and subsequent vessel leakage. concerning its effects on HSC/HPC localization within the fetal liver or their subsequent mobilization potential. However, lentiviral overproduction of Cxcr4 in adult hematopoietic cells resulted in an increased sensitivity to low levels of Sdf- $1 \alpha$ and altered mobilization in vitro. ${ }^{40}$ In addition, $\mathrm{cKit}^{+}$cells from both $\mathrm{Zeb} 2^{-/ \Delta \mathrm{Tie} 2 \mathrm{cre}}$ and Zeb2 ${ }^{-/ \Delta V a v-i C r e}$ fetal livers showed in vitro a significantly increased migration toward Sdf- $1 \alpha$, especially toward lower concentrations of this cytokine. We therefore propose that deletion of Zeb2 induces strong increases in Cxcr4 levels in HSCs/HPCs, making them highly sensitive to the low Sdf- $1 \alpha$ concentrations produced within the fetal liver, compromising their mobility and potential to enter the circulation and colonize the bone marrow. In the conventional Zeb2 knockout, no defects were seen in the formation of the cranial neural crest, but delamination and directional collective migration were significantly delayed just before embryonic lethality. ${ }^{15}$ Because the Sdf- $1 \alpha / \mathrm{Cxcr} 4$ axis is required for neural crest migration in vivo, and because it links chemotactic signals to migration and (de)adhesion, ${ }^{41}$ similar changes may underlie this neural crest phenotype in this mouse model. In conclusion, these findings suggest that in the Zeb2-knockout embryos, the increased levels of $\beta 1$ integrin and Cxcr4 may be responsible for the retention of the $\mathrm{Lin}^{-} \mathrm{CKit}^{+}$cells in the fetal liver, the decreased circulating progenitors, and, consequently, decreased bone marrow homing.

The decreased pool of circulating Ang1-expressing progenitor cells may also be involved in the observed bleeding phenotype and lethality. Most probably, the decreased Ang $1^{+}$-expressing cells in the circulation leads to the observed defects in pericyte recruitment, vessel instability, and enhanced leakage, as has been postulated for Fli1 and Runx1 knockouts in the mouse. ${ }^{30,31}$ However, this hypothesis is only based on correlations and remains to be functionally proven. The variable heart defects seen in the Zeb2 mutants may also be attributed to altered Ang1/Tie2 signaling, 
because severe cardiac defects have also been reported for Ang1and Tie2-deficient embryos. ${ }^{43,44}$ The reason for the specific localization of the intracranial bleeding, occurring mainly in neural tissues and especially in ganglia and ganglionic eminences, is unclear. However, it was reported previously that in both humans and mice, these regions are the most sensitive to vessel leakage as a consequence of pericyte recruitment defects. ${ }^{42}$

In the present study, we investigated the role of Zeb2 in hematopoiesis. To our knowledge, this is the first study addressing the in vivo role of Zeb family members in HSC/HPC biology. Conditional deletion of Zeb2 in the hematopoietic system results in embryonic lethality. No defect in hematopoietic cluster formation in the AGM region was found, but a block in early HSC/HPC differentiation was observed, suggesting an essential role for Zeb2 in differentiation potential, cell fate decisions, and/or stemness, rather than in the formation of HSCs/HPCs. In addition, aberrant HSC/HPC adhesion was observed, leading to decreased Ang1expressing progenitors in the embryonic circulation, thereby contributing to defective vessel maturation and bleeding. These results point to a key role for Zeb2 in embryonic hematopoiesis and set the stage for analyzing its possible role in pathophysiologic hematopoietic settings in the adult. Interestingly, over the last few years, various independent genome-wide retroviral insertional mutagenesis screens have identified Zeb2 as a possible gene involved in mouse B-cell lymphoma progression. ${ }^{46,47}$ In addition, altered Zeb2 expression was found to be correlated with increased leukemia progression in CALM-AF10-transgenic mice. ${ }^{48}$ However, in vivo evidence showing that changes in Zeb2 expression or function are directly involved in leukemia formation or progression is currently lacking. On the basis of our data, we propose that patients with Mowat-Wilson syndrome (recently shown to be caused by mutations in the gene encoding $Z E B 2^{49,50}$ ) may be predisposed to malignant blood cell disorders. Therefore, we propose adding screenings for hematologic abnormalities in the clinical management of this recently described genetic disorder.

\section{Acknowledgments}

We thank Daisy Ginneberge for her help with FACS cell sorting, An Zwijsen for her help with setting up the initial mice breeding and advice on the analysis of the gross phenotype of the mutant mouse embryos, and Griet Verstappen for providing Zeb2-null mouse ES cells.

This work was partially supported by the Association for International Cancer Research (grant 06-570 to J.J.H. and G.B.), by the Belgium Federation Against Cancer (Stichting tegen Kanker grant 203-2008 to J.J.H.), by Ghent University Methusalem (grant BOF09/01M00709), by the European Hematology Association (EHA Young Investigator Fellowship 2009/26 to S.G.), and by the German Research Foundation (Deutsche Forschungsgemeinschaft grant JA 1967/3-1 to V.J.). S.G. is a postdoctoral fellow of the Basic Science Research Foundation-Flanders (FWO).

\section{Authorship}

Contribution: S.G. and J.J.H. designed research; S.G., V.J., S.B., T.Y., B.D., M.C., K.H., E.S., T.R., L.H., and P.B. performed research; S.G., V.J., T.Y., B.D., D.H., and J.J.H. analyzed data; S.G. and J.J.H. wrote the manuscript with help from V.J., E.D., and D.H.; and L.U., G.B., E.D., D.H., and V.J. provided essential mouse and ES cell lines and/or infrastructural support.

Conflict-of-interest disclosure: The authors declare no competing financial interests.

The current affiliation of T.Y. is Cancer Science Institute of Singapore, National University of Singapore, Singapore.

Correspondence: Dr Jody J. Haigh, VIB, Department for Molecular Biomedical Research, Vascular Cell Biology Unit, Technologiepark 927, B-9052 Ghent (Zwijnaarde), Belgium; email: jody.haigh@dmbr.vib-UGent.be.

\section{References}

1. Postigo AA, Dean DC. Differential expression and function of members of the zfh- 1 family of zinc finger/homeodomain repressors. Proc Natl Acad Sci U S A. 2000;97(12):6391-6396.

2. Verschueren K, Remacle JE, Collart C, et al. SIP1, a novel zinc finger/homeodomain repressor, interacts with Smad proteins and binds to 5 '-CACCT sequences in candidate target genes. J Biol Chem. 1999;274(29):20489-20498.

3. Vandewalle C, Van Roy F, Berx G. The role of the ZEB family of transcription factors in development and disease. Cell Mol Life Sci. 2009;66(5):773787.

4. Kalluri R, Weinberg RA. The basics of epithelialmesenchymal transition. J Clin Invest. 2009; 119(6):1420-1428

5. Comijn J, Berx G, Vermassen P, et al. The twohanded $E$ box binding zinc finger protein SIP1 downregulates E-cadherin and induces invasion Mol Cell. 2001;7(6):1267-1278.

6. Schatton T, Frank NY, Frank MH. Identification and targeting of cancer stem cells. BioEssays. 2009;31(10):1038-1049.

7. Mani SA, Guo W, Liao MJ, et al. The epithelialmesenchymal transition generates cells with properties of stem cells. Cell. 2008;133(4):704715.

8. Gregory PA, Bert AG, Paterson EL, et al. The miR-200 family and miR-205 regulate epithelial to mesenchymal transition by targeting ZEB1 and SIP1. Nat Cell Biol. 2008;10(5):593-601.

9. Wellner U, Schubert J, Burk UC, et al. The EMT- activator ZEB1 promotes tumorigenicity by repressing stemness-inhibiting microRNAs. Nat Cell Biol. 2009;11(12):1487-1495.

10. Miranda-Saavedra D, Gottgens B. Transcriptional regulatory networks in haematopoiesis. Curr Opin Genet Dev. 2008;18(6):530-535.

11. Dzierzak E, Speck NA. Of lineage and legacy: the development of mammalian hematopoietic stem cells. Nat Immun. 2008;9(2):129-136.

12. de Bruijn MF, Ma X, Robin C, Ottersbach K, Sanchez MJ, Dzierzak E. Hematopoietic stem cells localize to the endothelial cell layer in the midgestation mouse aorta. Immunity. 2002;16(5): 673-683.

13. Boisset JC, van Cappellen W, Andrieu-Soler C, Galjart N, Dzierzak E, Robin C. In vivo imaging of haematopoietic cells emerging from the mouse aortic endothelium. Nature. 2010;464(7285):116120.

14. Higashi Y, Maruhashi M, Nelles L, et al. Generation of the floxed allele of the SIP1 (Smad-interacting protein 1) gene for Cre-mediated conditional knockout in the mouse. Genesis. 2002; 32(2):82-84.

15. Van de Putte T, Maruhashi M, Francis A, et al. Mice lacking ZFHX1B, the gene that codes for Smad-interacting protein-1, reveal a role for multiple neural crest cell defects in the etiology of Hirschsprung disease-mental retardation syndrome. Am J Hum Genet. 2003;72(2):465-470.

16. Chen MJ, Yokomizo T, Zeigler BM, Dzierzak E, Speck NA. Runx1 is required for the endothelial to haematopoietic cell transition but not thereafter. Nature. 2009;457(7231):887-891.

17. Maes C, Goossens S, Bartunkova S, et al. Increased skeletal VEGF enhances beta-catenin activity and results in excessively ossified bones. EMBO J. 2010;29(2):424-441.

18. Seuntjens E, Nityanandam A, Miquelajauregui A et al. Sip1 regulates sequential fate decisions by feedback signaling from postmitotic neurons to progenitors. Nat Neurosci. 2009;12(11):13731380

19. Bassez G, Camand OJ, Cacheux V, et al. Pleiotropic and diverse expression of ZFHX1B gene transcripts during mouse and human development supports the various clinical manifestations of the "Mowat-Wilson" syndrome. Neurobiol Dis. 2004;15(2):240-250.

20. Nelles L, Van de Putte T, van Grunsven L, Huylebroeck D, Verschueren K. Organization of the mouse Zfhx1b gene encoding the two-handed zinc finger repressor Smad-interacting protein-1. Genomics. 2003;82(4):460-469.

21. Osawa M, Hanada K, Hamada $H$, Nakauchi $H$. Long-term lymphohematopoietic reconstitution by a single CD34-low/negative hematopoietic stem cell. Science. 1996;273(5272):242-245.

22. Kisanuki YY, Hammer RE, Miyazaki J, Williams SC, Richardson JA, Yanagisawa M. Tie2-Cre transgenic mice: a new model for endothelial celllineage analysis in vivo. Dev Biol. 2001;230(2): 230-242.

23. Hsu HC, Ema H, Osawa M, Nakamura Y, Suda T, 
Nakauchi H. Hematopoietic stem cells express Tie-2 receptor in the murine fetal liver. Blood. 2000;96(12):3757-3762.

24. Soriano P. Generalized lacZ expression with the ROSA26 Cre reporter strain. Nat Genet. 1999; 21(1):70-71.

25. Okuda T, van Deursen J, Hiebert SW, Grosveld G, Downing JR. AML1, the target of multiple chromosomal translocations in human leukemia, is essential for normal fetal liver hematopoiesis. Cell. 1996;84(2):321-330.

26. Spyropoulos DD, Pharr PN, Lavenburg KR, et al. Hemorrhage, impaired hematopoiesis, and lethality in mouse embryos carrying a targeted disruption of the Fli1 transcription factor. Mol Cell Biol. 2000;20(15):5643-5652.

27. Pandolfi PP, Roth ME, Karis A, et al. Targeted disruption of the GATA3 gene causes severe abnormalities in the nervous system and in fetal liver haematopoiesis. Nat Genet. 1995;11(1):40-44

28. de Boer J, Williams A, Skavdis G, et al. Transgenic mice with hematopoietic and lymphoid specific expression of Cre. Eur J Immunol. 2003; 33(2):314-325.

29. $\mathrm{He} \mathrm{C}, \mathrm{Hu} \mathrm{H}$, Braren $\mathrm{R}$, et al. c-myc in the hematopoietic lineage is crucial for its angiogenic function in the mouse embryo. Development. 2008; 135(14):2467-2477.

30. Takakura N, Watanabe T, Suenobu S, et al. A role for hematopoietic stem cells in promoting angiogenesis. Cell. 2000;102(2):199-209.

31. Thurston G, Suri C, Smith K, et al. Leakage-resistant blood vessels in mice transgenically overexpressing angiopoietin-1. Science. 1999; 286(5449):2511-2514.

32. Verstappen G, van Grunsven LA, Michiels C, et al. Atypical Mowat-Wilson patient confirms the importance of the novel association between ZFHX1B/SIP1 and NuRD corepressor complex. Hum Mol Genet. 2008;17(8):1175-1183.
33. Hirsch E, Iglesias A, Potocnik AJ, Hartmann U, Fassler R. Impaired migration but not differentiation of haematopoietic stem cells in the absence of beta1 integrins. Nature. 1996;380(6570):171175.

34. Papayannopoulou T, Priestley GV, Nakamoto B, Zafiropoulos V, Scott LM. Molecular pathways in bone marrow homing: dominant role of alpha(4)beta(1) over beta(2)-integrins and selectins. Blood. 2001;98(8):2403-2411.

35. Lapidot $\mathrm{T}$, Kollet $\mathrm{O}$. The essential roles of the chemokine SDF-1 and its receptor CXCR4 in human stem cell homing and repopulation of transplanted immune-deficient NOD/SCID and NOD/ SCID/B2m(null) mice. Leukemia. 2002;16(10): 1992-2003.

36. Sweeney EA, Lortat-Jacob H, Priestley GV Nakamoto B, Papayannopoulou T. Sulfated polysaccharides increase plasma levels of SDF-1 in monkeys and mice: involvement in mobilization of stem/progenitor cells. Blood. 2002;99(1):44-51.

37. Peled A, Grabovsky V, Habler L, et al. The chemokine SDF-1 stimulates integrin-mediated arrest of CD34(+) cells on vascular endothelium under shear flow. J Clin Invest. 1999;104(9): 1199-1211.

38. Hattori $\mathrm{Y}$, Kato H, Nitta M, Takamoto S. Decrease of L-selectin expression on human CD34+ cells on freeze-thawing and rapid recovery with shortterm incubation. Exp Hematol. 2001;29(1):114122.

39. Liles WC, Broxmeyer HE, Rodger E, et al. Mobilization of hematopoietic progenitor cells in healthy volunteers by AMD3100, a CXCR4 antagonist. Blood. 2003;102(8):2728-2730.

40. Kahn J, Byk T, Jansson-Sjostrand L, et al. Overexpression of CXCR4 on human CD34+ progenitors increases their proliferation, migration, and NOD/SCID repopulation. Blood. 2004;103(8): 2942-2949.
41. Theveneau E, Marchant L, Kuriyama S, et al. Collective chemotaxis requires contact-dependent cell polarity. Dev Cell. 2010;19(1):39-53.

42. Hart A, Melet F, Grossfeld P, et al. Fli-1 is required for murine vascular and megakaryocytic development and is hemizygously deleted in patients with thrombocytopenia. Immunity. 2000;13(2):167177.

43. Sato TN, Tozawa Y, Deutsch U, et al. Distinct roles of the receptor tyrosine kinases Tie-1 and Tie-2 in blood vessel formation. Nature. 1995; 376(6535):70-74.

44. Suri C, Jones PF, Patan S, et al. Requisite role of angiopoietin-1, a ligand for the TIE2 receptor, during embryonic angiogenesis. Cell. 1996;87(7): 1171-1180.

45. Braun $\mathrm{A}, \mathrm{Xu} \mathrm{H}, \mathrm{Hu} F$, et al. Paucity of pericytes in germinal matrix vasculature of premature infants. J Neurosci. 2007;27(44):12012-12024.

46. Mikkers $\mathrm{H}$, Allen J, Knipscheer $\mathrm{P}$, et al. Highthroughput retroviral tagging to identify components of specific signaling pathways in cancer. Nat Genet. 2002;32(1):153-159.

47. Lund AH, Turner G, Trubetskoy A, et al. Genomewide retroviral insertional tagging of genes involved in cancer in Cdkn2a-deficient mice. Nat Genet. 2002;32(1):160-165.

48. Caudell D, Harper DP, Novak RL, et al. Retroviral insertional mutagenesis identifies Zeb2 activation as a novel leukemogenic collaborating event in CALM-AF10 transgenic mice. Blood. 2010; 115(6):1194-1203.

49. Van de Putte T, Francis A, Nelles L, van Grunsven LA, Huylebroeck D. Neural crest-specific removal of Zfhx1b in mouse leads to a wide range of neurocristopathies reminiscent of Mowat-Wilson syndrome. Hum Mol Genet. 2007;16(12):1423-1436.

50. Mowat DR, Wilson MJ, Goossens M. MowatWilson syndrome. J Med Genet. 2003;40(5): 305-310. 